\title{
Application of fourier transform infrared spectroscopy for authentication of functional food oils.
}

\begin{abstract}
In recent years, the authentication of edible fats and oils has become an important issue for food producers, consumers, and regulatory authorities due to religious and economic reasons. Some highpriced edible oils such as extra virgin olive oil (EVOO), virgin coconut oil (VCO), and cod liver oil (CLO) are adulterated with lower price oils to improve profits. These oils can be considered functional food oils due to their beneficial effects on human health. Fourier transform infrared (FTIR) spectroscopy combined with suitable chemometrics techniques has emerged as a potential tool that allows analysts to authenticate high-value edible oils. This review describes the potential use of FTIR spectroscopy for authentication of three functional food oils, namely, EVOO, VCO, and CLO.
\end{abstract}

Keyword: FTIR spectroscopy; Chemometrics; Authentication; Olive oil; Virgin coconut oil; Cod liver oil. 